\title{
CORRECTION AND SUPPLEMENT TO THE PAPER THE DIRECT PRODUCT OF RIGHT SINGULAR SEMIGROUPS AND CERTAIN GROUPOIDS ${ }^{1}$
}

T. TAMURA, R. B. MERKEL AND J. F. LATIMER

First we would like to correct a few places in the paper as follows: p. 122, 2nd line from the bottom (condition P5.5), next to "two-sided identity," add

$$
\text { " } e_{\alpha} \text { and } e_{\alpha} e_{\beta}=e_{\beta} . "
$$

p. 123,1 st line,

$$
\text { delete "P5.3". }
$$

p. 123, 2nd line from the bottom (the proof of Theorem 6)

$$
\text { "P5.3" is replaced by "P5.5". }
$$

To make sure, we shall restate the changed part:

P5.5. There is a decomposition $\left\{S_{\alpha}\right\}$ of $S$ such that each $S_{\alpha}$ is a groupoid with a two-sided identity $e_{\alpha}$ and $e_{\alpha} e_{\beta}=e_{\beta}$.

VIII $_{2}^{\prime}$. $\{$ P5.1, P5.2, P5.5 $\}$.

Theorem 6 can be restated as follows:

Theorem 6. A groupoid $S$ is an $M$-groupoid if and only if $S$ is a right zero band of groupoids $S_{\alpha}$ with identity $e_{\alpha}, S=\cup_{\alpha \in R} S_{\alpha}, S_{\alpha} S_{\beta} \subset S_{\beta}$ such that $e_{\alpha} e_{\beta}=e_{\beta}$.

In other words:

Let $S$ be a right zero band of groupoids $S_{\alpha}$ with an identity $e_{\alpha}$. Then $S$ is the direct product of a right zero band and a groupoid with an identity if and only if

$$
e_{\alpha} e_{\beta}=e_{\beta} .
$$

Let $\mathrm{VIII}_{3}^{\prime}$ denote the original $\mathrm{VIII}_{2}^{\prime}$, that is, $\mathrm{VIII}_{3}^{\prime} \cdot\{$ P5.1, P5.2, P5.3 with the original P5.5 $\}$.

$\mathrm{VIII}_{3}^{\prime}$ is a sufficient condition for $S$ to be an $M$-groupoid, but not a necessary condition.

We thank Professor Reikichi Yoshida for his remark.

Received by the editors March 22, 1965.

${ }^{1}$ Proc. Amer. Math. Soc. 14 (1963), 118-123. 